\title{
Paediatric gastrointestinal stromal tumours arising from the stomach: a clinicopathologic review of 85
}

\section{cases}

\begin{abstract}
Aim of the study: Gastrointestinal stromal tumors (GISTs) may now be considered to encompass mesenchymal tumors that express Kit protein (CD117) or have activating mutations of receptor tyrosine kinase genes (PDGFRA). The purpose of this study is to define clinicopathologic characteristics of gastric GISTs in children.
\end{abstract}

Materials and Methods: We conducted a review of the literature from 1962 and enrolled 85 patients (younger than 16 years of age) with gastric GISTs, including two cases treated in our pediatric surgery unit. All the patients' clinical data and follow-up information were reviewed, including age, gender, tumour size and localization, presence of Kit mutation and outcome.

Case 1: A 16-year-old girl with a history of weakness and pallor. A computer tomography scan confirmed 4 gastric lesions. The lesion in the fundus was excised and an inferior-middle gastric resection was perfomed. At 3 years follow-up the patient presents a progression of liver metastasis.

Case 2: A 13-year-old boy was referred to our pediatric surgery unit because of an ulcerated neoformation of 3-cm-sized located in the gastric antrum. The patient underwent laparotomy gastric wedge resection. At the follow-up of 9 years the patient was disease free.

Results: Our results are based on our systematic review of 85 cases. The multifocal disease was identified in 21 children (25\%). 10 patients are died, all patients presented liver metastases but only 1 had multiple nodules.

Conclusions: The majority of GISTs occur in girls that suggests a developmentally related sex difference. There are no relationship between multiple locations and worst prognosis. The presence of liver metastases is associated with a shorter survival.

Keywords: paediatric neoplasm, mesenchymal tumours, gastrointestinal stromal tumors, kit protein, platelet derived growth factor receptor alpha, interstitial cells of cajal
Volume I Issue 6 - 2014

\author{
Michele llari,' Fabiano Nino,' Andrea \\ Zangari, ${ }^{2}$ Giovanni Torino, ${ }^{3}$ Rosella Tallarico,' \\ Giovanni Cobellis,' Ascanio Martino' \\ 'Pediatric Surgery Unit, Salesi Children's Hospital, Italy \\ ${ }^{2}$ Department of Pediatric Surgery, San Camillo-Forlanini \\ Hospital, Italy \\ ${ }^{3}$ Surgical Unit of Pediatric Urology, Bambino Gesu Children's \\ Hospital, Italy
}

Correspondence: Fabiano Nino, Pediatric Surgery Unit, Sales Children $\square$ s Hospital,Via Corridoni, I I $\square$ Ancona, Italy, Tel +39 3478II5859, Fax +39 07I5962442

Email fabianonino80@gmail.com

Received: October 17,2014 | Published: November 06, 2014
Abbreviations: GIST: gastrointestinal stromal tumor, PDGFRA: platelet derived growth factor receptor alpha, GI: gastrointestinal, CD: cluster of differentiation, ICC: interstitial cells of cajal, GANTs: gastrointestinal autonomic nerve tumours, CT: computed tomography, LC: lesser curvature, HPFs: high power fields, NF1: neurofibromatosis type 1

\section{Introduction}

Gastrointestinal stromal tumours (GISTs) comprise the largest group of mesenchymal tumours of the digestive tract. Pediatric GIST is a rare entity, with clinical characteristics different from those of adults. The reports of this entity in pedaitric age are limited to case report or small series, so that the true incidence in children is unknown. Actually, GISTs can be considered as encompassing mesenchymal tumours that express Kit protein (CD117 in the standardized terminology of leukocyte antigens) or that have activating mutations of receptor tyrosine kinase genes [c-kit or platelet derived growth factor receptor alpha (PDGFRA)]. Kit expression is extremely important in the development of several cell types, including haematopoietic stem cells, mast cells, germ cells, melanocytes, some epithelial cells and the interstitial cells of Cajal. ${ }^{1}$ However, approximately $10-15 \%$ of GISTs do not express Kit, ${ }^{2}$ within this group, a large subset would have acquired PDGFRA function mutations [3]. Mutations in PDGFRA can represent an alternative mechanism in GIST oncogenesis. It has been suggested that GISTs originate from the ICCs, or from a primitive stem cell that differentiates towards both the ICC and smooth muscle phenotype. ${ }^{4,5}$ ICCs have a function as pacemaker cells in the gastrointestinal (GI) tract and they mediate impulses from the enteric nervous system to smooth muscle cells. ICCs express the Kit protein and inhibition of Kit function interferes with the autonomous movement of the GI tract. ${ }^{6-8}$ GISTs cover a spectrum that includes benign and malignant variants and they range in size from several millimeters to over $30 \mathrm{~cm}$. Most lesions are well circumscribed, but are not encapsulated and some are multi-nodular. ${ }^{9}$ Even though recent evidence supports the notion that gastrointestinal autonomic nerve tumours (GANTs) represent only a phenotypic variant of GISTs ${ }^{10}$ this study excluded known cases of GANT reviewed in literature. There are reports published on the extremely rare association between GIST and locoregional lymphonodal metastasis. ${ }^{11}$

\section{Material and methods}

We conducted a review of the literature from 1962 and enrolled 85 patients (younger than 16 years of age) with gastric GISTs, including two cases treated in our department. We reviewed all pediatric cases of gastric GISTs with particular attention to the presence of locoregional lymphonodal metastasis, as in our cases. All the patients' clinical data and follow-up information were reviewed, including age, gender, tumour size and localization, presence of Kit mutation and outcome. In fact, the criteria used to predict the behaviour of such tumours vary significantly with the site affected, the mitotic rate and tumour size. 


\section{Case I}

A 15-year-old girl with a history of weakness and pallor following a lipotimia episode was found to have anaemia (Hb $5.6 \mathrm{~g} / \mathrm{dl}$ ). She was treated with oral and intravenous iron supplementation for 3 months. Owing to the persistent severe anaemia, she underwent further examination for GI bleeding. A Meckel's scan was performed which showed a mild focus of uptake in the mesogastrium. Abdominal ultrasound revealed two hypoechoic 3-cm-sized ovoid lesions of the anterior wall of the stomach, with both central and peripheral vascularization. An upper gastrointestinal endoscopy was performed which showed dark red blood in the stomach and 4 nodular lesions: 3 were located in the antrum and 1 in the fundus presented ulceration. A computed tomography (CT) scan confirmed the 4 lesions originating in the stomach wall: the biggest lesion was located in the iuxta cardial area $(3.2 \mathrm{~cm}), 2$ lesions were located in the antrum $(3.1$ and $2.3 \mathrm{~cm}$ respectively), and the smallest lesion $(0.8 \mathrm{~cm})$ was located in the posterior wall of the antrum along the greater curvature. The patient was transferred to surgery for the removal of the masses. We performed a segmental gastric resection by laparotomy. During the surgery, 4 soft ovoid masses were found in the stomach. The lesion in the fundus was excised and an inferior-middle gastric resection was performed. There were no perioperative complications and she was discharged on postoperative day 12 . The final pathological finding of this specimen was multifocal gastrointestinal stromal tumour (GISTs), all the lesions had negative resection margins and histology showed 50 mitotic figures per 50 high power fields. At 12 month follow-up, a hepatic metastasis was observed and the patient was treated with chemotherapy for 2 years. At 3 years follow-up, the patient presented a progression of liver metastases.

\section{Case 2}

A 13-year-old boy was referred to our pediatric surgery unit with an ulcerated neoformation of $3.0 \mathrm{~cm}$ located in the antrum of the stomach. This had been detected with an upper gastrointestinal endoscopy performed because of a history of weakness, pallor and anaemia $(\mathrm{Hb} 7,8 \mathrm{~g} / \mathrm{dl})$. Physical examination revealed the boy to be in good condition with no palpable lymphadenopathy or abdominal masses. A repeated esophagogastroscopy confirmed the presence of a gastric wall-mass, with ulcerations, in the posterior wall of the antrum. Ultrasound evaluation showed a solid and homogeneous mass of $41 \times 21 \mathrm{~mm}$. The patient underwent laparotomy gastric wedge resection and a pyloroplasty was performed. Recovery was uneventful, the boy resumed normal diet on day 7 and was discharged on postoperative day 10 . The final pathology results showed a $3.0 \mathrm{~cm}$ diameter tumour with central mucosal ulceration extending from the mucosa to the muscularis propria, 8/10 mitotic figures per high power field. The results of immunohistochemical analysis revealed actin positive, desmin and S-100-negative, findings consistent with a diagnosis of stromal tumour. At follow-up of 9 years, the patient was disease free.

\section{Results}

We analyzed gastric GISTs presented in 20 males and 65 females, the youngest of whom were a 3.5-year-old girl and a 4-yearold boy. Male to female ratio of approximately 1:3, 3 suggests a developmentally related sex difference. ${ }^{12}$ The greater number of patients with a known clinical history presented gastrointestinal bleeding (68/85 patients, $80 \%)$. The bleeding was most commonly insidious, leading to anaemia and related symptoms such as syncope and weakness.

In our review, a preponderance of the tumours with specified location occurred in the lesser curvature (LC) $(n=24), 28$ patients
(33\%) developed recurrences in the liver and/or in the peritoneal cavity and 10 patients of these died during the follow-up. In these 28 patients, the tumours measured from 3 to $35 \mathrm{~cm}$ and only 1 patient had multiple nodules, no specified location was identified and only five patients $(18 \%)$ showed high mitotic activity that varied from 0 to 250/50 high power fields (HPFs) (median 6/50). Multifocal disease was identified in 21 children $(25 \%)$ and this findings raises the question of a possible relationship with other syndromes typically associated with multiple GISTs, such as familial GIST syndrome, Carney's triad and neurofibromatosis type 1 (NF1), ${ }^{11}$ of these, the only patient that died presented liver metastases. All the data regarding our two cases above and the 83 cases previously published in the literature are summarized in Table 1 (Included as supplementary).

\section{Discussion}

Gastrointestinal stromal tumours occur in the mesenchymal tissue of the alimentary tract and affect predominantly middle-aged or older patients. These tumours can arise anywhere in the gastrointestinal tract, but frequently, they are located in the stomach $(30-70 \%)$ and the small bowel (20-40\%). ${ }^{13}$ Gastric GISTs are rare in children and include a spectrum of clinically benign and malignant tumours. Actually there are not available guidelines of pediatric GIST, and the recommendations are based on the management of adult patients.

It is extremely important to note that there is no relationship in pediatric literature between multiple gastric locations and the worst prognosis and the association between gastric GIST and locoregional lymphonodal metastasis is rarely described. ${ }^{11}$ Patients with familial GISTs have inherited germline mutations in Kit or PDGFRA, ${ }^{14,15}$ but the pathogenesis of GIST in the two latter syndromes is unknown. GISTs in patients with NF1 have recently been shown to lack the Kit mutation. ${ }^{16}$ GISTs associated with Carney's triad typically occur in a younger age group with female predominance and include one or two additional tumours, such as extra-adrenal paraganglioma or pulmonary chondroma. ${ }^{11}$ Prognostically, gastric GISTs in children are heterogeneous. A majority of these tumours are clinically indolent with an excellent long-term prognosis. Gastric GISTs are classified, according to size and mitotic count, into three categories: benign, malignant and uncertain or low malignant potential (Table 2).

he presence of peritoneal or liver metastases at presentation is an adverse prognostic factor and is associated with a shorter survival, ${ }^{17}$ and the subset of GISTs that has a high likelihood of malignant behaviour is generally identified by increased tumour size and mitotic activity in the context of tumour location. ${ }^{18,19}$

\section{Management}

The therapeutic approach is stage related, the main treatment for localized GISTs, is gross surgical resection with preservation of intact pseudo capsules. ${ }^{20-23}$ The abdomen should be thoroughly explored by laparotomy, with close examination of the peritoneal surfaces and liver to identify metastases. In the case of adherent GIST, resection of adjacent organs should be performed to obtain negative margins, removal of uninvolved tissue is not recommended. Lymphadenectomy is necessary only in the presence of evident nodal involvement (rare in GISTs). ${ }^{24}$ Laparoscopy can be used to resect small to intermediate sized tumours, especially from the stomach. This approach decreases morbidity, shortens hospital stay and has local recurrence rates similar to open procedure. ${ }^{25,26}$ For lesions of indeterminate type, endoscopic techniques, including fine needle aspiration or biopsy, are the methods of choice, ${ }^{27}$ percutaneous biopsy is not recommended because of tumour rupture and/or peritoneal spread. In the pathogenesis of GISTs, mutation of the Kit gene leads to dysregulated signalling 
of the Kit tyrosine kinase receptor, which is involved in critical cellular pathways that control cell proliferation and differentiation. This is the reason why, in advanced disease, competitive inhibition of monoclonal antibodies prevents the transfer of phosphate groups from ATP to tyrosine residues on substrate protein (phosphorylation), thereby interrupting proliferative intracellular signalling pathways. ${ }^{28}$ Imatinib is a small molecule tyrosine kinase inhibitor with activity against Kit and PDGFRA, it is widely used in the treatment of pediatric gastric GISTs owing to two important findings that suggest that Imatinib might be effective against such lesions. Imatinib can inhibit the kinase activity of both wild-type and mutant Kit, ${ }^{29}$ and it has been shown to inhibit the growth of a GIST cell line containing a Kit gene mutation..$^{30}$ Imatinib reliably achieves disease control in 70 $85 \%$ of patients with advanced GISTs and median progression-free survival is in the range of 20-24months. Although Imatinib has greatly improved the quality of life and survival of patients with advanced gastric GISTs, most patients are not cured by this treatment. Sunitinib has been approved for the treatment of advanced GISTs in patients who fail (or are intolerant to) Imatinib therapy. In addition to targeting Kit, Sunitinib also has antiangiogenic effects through the inhibition of vascular endothelial growth factor receptors.

\section{Conclusion}

Gastric GISTs encompass a spectrum of clinically benign and malignant tumours, the overall survivor rate of paediatric gastric GISTs is $88.8 \%$. Multifocal gastric GIST is not associated with the worst prognosis or with a high risk of local recurrence. Therefore, organ sparing surgery should be considered also in multifocal disease, even in the presence of regional lymphonodal metastasis. The presence of liver or/and peritoneal metastasis is an adverse prognostic factor associated with a shorter survival. However, the treatment and prognosis of children with gastric GISTs, has been substantially changed by the discovery of oncogenic kinase mutations in the vast majority of these tumours and the introduction of molecular therapies that inhibit such molecular defects. Moreover, one important question remains unanswered: our first case shows that a patient with multifocal tumor treated only with surgery can, in a long term period, develop metastasis, therefore it would be interesting to investigate the outcome of the patients affected by mutifocal tumor managed by a combined therapy (surgical treatment plus adjuvant chemotherapy). Several trials are investigating the role of adjuvant therapy in the management of patients with completely resected gastric GISTs. ${ }^{21} \mathrm{~A}$ further subject of active investigation is the use of neoadjuvant imatinib to downsize primary or metastatic disease before surgery ${ }^{31}$

\section{Acknowledgements}

None.

\section{Conflicts of Interest}

There is no conflict of interest.

\section{Funding}

None.

\section{References}

1. Vliagoftis $\mathrm{H}$, Worobec AS, Metcalfe DD. The protooncogene c-kit and c-kit ligand in human disease. J Allergy Clin Immunol. 1997;100(4): 435-440.

2. Rychter MD, Wasag B, Stul M, et al. Gastrointestinal stromal tumours (GISTs) negative for KIT (CD117 antigen) immunoreactivity. J Pathol. 2004;202 (4): 430-438.
3. Heinrich MC, Corless CL, Demetri GD, et al. Kinase mutations and Imatinib response in patients with metastatic gastrointestinal stromal tumor. J Clin Oncol. 2003;21(23): 4342-4349.

4. Rubin BP, Fletcher JA, Fletcher CD. Molecular Insights into the Histogenesis and Pathogenesis of gastrointestinal Stromal Tumors. Int J Surg Pathol. 2000;8(1): 5-10.

5. Kindblom LG, Remotti HE, Aldenborg F, et al. Gastrointestinal pacemaker cell tumor (GIPACT): gastrointestinal stromal tumors show phenotypic characteristics of the interstitial cells of Cajal. Am J Pathol. 1998;152(5):1259-1269.

6. Maeda $\mathrm{H}$, Yamagata A, Nishikawa $\mathrm{S}$, et al. Requirement of c-kit for development of intestinal pacemaker system. Development. 1992;116(2): 369-375

7. Ward SM, Burns AJ, Torihashi S, et al. Mutation of the proto-oncogene c-kit blocks development of interstitial cells and electrical rhythmicity in murine intestine. J Physiol. 1994;480 (Pt 1): 91-97.

8. Huizinga JD, Thuneberg L, Kluppel M, et al. W/kit gene required for interstitial cells of Cajal and for intestinal pacemaker activity. Nature. 1995;373(6512): 347-349.

9. Sabah M, Leader M, Kay E, et al. Gastrointestinal stromal tumours: An update. Current Diagnostic Pathology. 2005;11(6): 400-410.

10. Segal A, Carello S, Caterina P, et al. Gastrointestinal autonomic nerve tumors: a clinicopathological, immunohistochemical and ultrastructural study of 10 cases. Pathology. 1994;26(4):439-447.

11. Prakash S, Sarran L, Socci N, et al. Gastrointestinal stromal tumors in children and young adults: a clinicopathologic, molecular, and genomic study of 15 cases and review of the literature. J Pediatr Hematol Oncol. 2005;27(4): 179-187.

12. Miettinen M, Lasota J, Sobin HL. Gastrointestinal stromal tumors of the stomach in children and young adults. A clinicopathologic, immunohistochemical, and molecular genetic study of 44 cases with long-term follow-up and review of the literature. Am J Surg Pathol. 2005;29(10): 1373-1381.

13. Kuroiwa M, Hiwatari M, Hirato, et al. Advanced-stage gastrointestinal stromal tumor treated with Imatinib in a 12-year-old girl with a unique mutation of PDGFRA. J Pediatr Surg. 2005;40(11):1798-1801.

14. Hirota S, Okazaki T, Kitamura Y, et al. Cause of familial and multiple gastrointestinal autonomic nerve tumors with hyperplasia of interstitial cells of Cajal is germline mutation of the c-kit gene. Am J Surg Pathol. 2000;24(2): 326-327.

15. Chompret A, Kannengiesser C, Barrois M, Terrier P, Dahan P, et al. (2004) PDGFRA germline mutation in a family with multiple cases of gastrointestinal stromal tumor. Gastroenterology. 2004; 126(1): 318321.

16. Kinoshita K, Hirota S, Isozaki K, et al. Absence of c-kit gene mutations in gastrointestinal stromal tumours from neurofibromatosis type 1 patients. J Pathol. 2004;202(1): 80-85.

17. Dematteo RP, Lewis JJ, Leung D, et al. Two hundred gastrointestinal stromal tumors: recurrence patterns and prognostic factors for survival. Ann Surg. 2000;231(1): 51-58.

18. Fletcher CD, Berman JJ, Corless C, et al. Diagnosis of gastrointestinal stromal tumors: a consensus approach. Hum Pathol. 2002;33(5): 459465.

19. Miettinen M, El Rifai W, Sobin HL, et al. Evaluation of malignancy and prognosis of gastrointestinal stromal tumors: a review. Hum Pathol. 2002;33(5):478-483.

20. Blay JY, Bonvalot S, Casali P, et al. Consensus meeting for the management of gastrointestinal stromal tumors. Report of the GIST consensus conference of 20-21 March 2004, under the auspices of European Society for Medical Oncology. Ann Oncol. 2005;16(4): 566578 
21. Dematteo RP, Heinrich MC, El-Rifai WM, et al. Clinical management of gastrointestinal stromal tumor (GIST) before STI-571. Hum Pathol. 2002; 33(5): 466-477.

22. Demetri G, Benjamin R, Blanke CD, et al. NCCN Task Force report: optimal management of patients with gastrointestinal stromal tumor (GIST) - expansion and update of NCCN Clinical Practice Guidelines. J Natl Compr Canc Netw . 2004;2(suppl 1): S1-S26.

23. Eisemberg BI, Judson I. Surgery and Imatinib in the management of GIST: emerging approaches to adjuvant and neoadjuvant therapy. Ann Surg Oncol. 2004;11(5):465-475.

24. Rubin BP, Heinrich MC, Corless CL, et al. Gastrointestinal stromal tumour. Lancet. 2007;369(9574):1731-1741.

25. Bedard EL, Mamazza J, Schlachta CM, et al. Laparoscopic resection of gastrointestinal stromal tumors: not all tumors are created equal. Surg Endosc. 2006;20(3): 500-503.

26. Novitsky YW, Kercher KW, Sing RF, et al. Long-term outcomes of laparoscopic resection of gastric gastrointestinal stromal tumors. Ann Surg. 2006;243(6):738-745.
27. von Mehren M, Watson JC. Gastrointestinal stromal tumors. Hematol Oncol Clin North Am. 2005;19(3): 547-564.

28. Joensuu H, Fletcher C, Dimitrijevic S, et al. Management of malignant gastrointestinal stromal tumours. Lancet Oncol. 2002;3(11): 655-664.

29. Heinrich MC, Blanke CD,et al. Inhibition of KIT tyrosine kinase activity: a novel molecular approach to the treatment of KIT-positive malignancies. J Clin Oncol. 2002;20(6):1692-1703.

30. Tuveson DA, Willis NA, Jacks T, et al. STI571 inactivation of the gastrointestinal stromal tumor c-Kit oncoprotein: biological and clinical implications. Oncogene. 2001; 20(36): 5054-5058.

31. Blackstein ME, Blay JY, Corless C, et al. Gastrointestinal stromal tumors: consensus statement on diagnosis and treatment. Can J Gastroenterol. 2006;20(3):157-163. 